\title{
El avance en la tecnología móvil y su impacto en la sociedad
}

The advance in mobile technology and its impact on society.

Efraín Velasteguí López. ${ }^{1}$ \& Barona López Gustavo²

\section{Resumen.}

El avance en la tecnología móvil es muy importante destacar que se ha mejorado la forma de comunicarnos ya que hoy en día existen los teléfonos de alta generación que cada día mejora sus nuevos modelos de dispositivos móviles confortantes para nuestra sociedad ya que nos permite estar comunicados de largas distancia en todo el mundo podemos decir que la tecnología móvil se ha destacado por buscar estar siempre comunicados con nuestro entorno.

Los dispositivos móviles son de gran ayuda en la vida del ser humano ya que como anteriormente mencionamos son unos dispositivos que tiene muchas características y funciones que ayudan en nuestro entorno como el tamaño porque es importante destacar lo importante que es el tamaño de este dispositivo ya que nos permite cargarle en la mano en el bolsillo del pantalón de la cartera etc.

La movilidad que también nos facilita es increíble y sobre todo la interacción que es lo que más destaca a este dispositivo es que nos permite estar interactuando con demás personas ya sea a través de mensajes llamadas en fin cabe destacar que también que este dispositivo tiene sus ventajas y desventajas y tenemos que hacer un buen uso de estas nuevas tecnologías que tenemos hoy en día.

Los estudios estadísticos también se ha mostrado que cada uno de los ecuatorianos cuenta con un celular inteligente estos les permite agilizar su entorno de estar comunicados.

La telefonía móvil se ha desempeñado por satisfacer cualquier necesidad del ser humano y es importante ya que podemos usarla ante cualquier emergencia.

Palabras claves: Alfabetismo, Digital, Adultos, tecnología, Aprendizaje.

\footnotetext{
${ }^{1}$ Ciencia digital, Ambato, Ecuador, luisefrainvelastegui@cienciadigital.org

${ }^{2}$ Escuela Politécnica Nacional, Facultad de Ingeniería Mecánica, Quito, Ecuador, barona_gustavo@ @otmail.com
} 


\begin{abstract}
.
The advance in mobile technology is very important to emphasize that the way we communicate has been improved since today there are high-generation phones that improve every day their new models of comforting mobile devices for our society as it allows us to be communicated from long distance around the world we can say that mobile technology has stood out for seeking to be always communicated with our environment.
\end{abstract}

Mobile devices are of great help in the life of the human being as we mentioned above are some devices that have many features and functions that help in our environment such as size because it is important to highlight how important the size of this device is. it allows us to carry it in the hand in the pocket of the pants of the wallet etc.

The mobility that also facilitates us is incredible and above all the interaction that is what stands out most to this device is that it allows us to be interacting with other people either through messages called in order to note that this device also has its advantages and disadvantages and we have to make good use of these new technologies that we have today.

Statistical studies have also shown that each of the Ecuadorians has an intelligent cell phone that allows them to streamline their environment of being communicated.

Mobile telephony has been able to satisfy any need of the human being and is important since we can use it in any emergency.

Keywords: Literacy, Digital, Adults, technology, Learning.

\title{
Introducción
}

Según los autores (Garita, 2013), (Weiss Muller, 2008), (Quijada, 2014), (Diseño, 2012) Definen que la tecnología móvil en sus inicios eran desarrolladas para utilizarse en las computadoras de escritorio y portátiles, lo común en ese tiempo sin embargo los dispositivos han cambiado y con ese cambio se ha presentado la evolución de la tecnología .ahora bajo esta tecnología los recursos son creados para ejecutarse mediante dispositivos móviles inteligentes.

En la actualidad los dispositivos móviles forman un grupo sumamente heterogéneo y pueden incorporar casi cualquier componente como hardware y software que amplíe y diversifique su función inicial.

Los dispositivos móviles pueden ser clasificados en los siguientes grupos:

- Los dispositivos de comunicación: son los dispositivos que presentan como función principal ofrecer una infraestructura de comunicación telefónica. Además, brindar la posibilidad de servicios como envió de mensajes de texto y multimedia. 
- Los dispositivos de comunicación: son los dispositivos que ofrecen mayores capacidades de procesamiento de datos y cuentan con una pantalla y teclado muy similares a la experiencia de los computadores personales. Los PDA y las computadoras portátiles tienen como sus principales beneficios la presentación de mayores y mejores servicios inclusive que los computadores de escritorio.

- Los dispositivos reproductores de multimedia: son aquellos que ha sido diseñados para proporcionar a los usuarios la oportunidad de reproducir uno o varios formatos digitales, ya sea audio, video, imágenes. Algunos tipos de dispositivos son los reproductores de MP3 y MP4, los DVD portátiles y todos los lectores de libro electrónicos.

El avance de la tecnología cada vez es más grande conforme los año que van pasando cada vez el mundo de la tecnología por esto nos mencionaba anteriormente que los dispositivos móviles han cambia mucho en torno a nuestras vidas ya que iniciamos con unos dispositivos grandes de tamaño y muy dificultoso realizar actividades que hoy en día podemos realizar en nuestro dispositivo inteligente, además de destacar el peso y tamaño de aquellos dispositivos haciendo una pequeña comparación podemos decir que el de hoy la telefonía móvil es más avanzada y fácil de usar en cualquier parte del mundo.

La telefonía móvil se ha evolucionado de una manera muy factible para toda la sociedad ya que hoy en día nos permite estar comunicados en cualquier parte del mundo, además de ser un dispositivo liviano le podemos llevar hasta en el bolsillo la gama de actividades que se pueden realizar en este dispositivo. Mucha característica nos mencionaba que son importantes al tener en cuenta ya que es muy cierto que estos dispositivos sirven para estar comunicados en todo momento ya sea una emergencia o por cualquier circunstancia, también es muy factible ya que podemos estar comunicados no solo por llamadas de vos sino que también por mensajes de texto, de correos electrónicos o a su vez a través de nuestras redes sociales.

Además, es un dispositivo que nos permite almacenar una cierta cantidad de datos y archivos que a su vez podemos realizar en nuestros dispositivos o a su vez descárgalos y tener archivos importantes guardados en el dispositivo ya que hoy con estos dispositivos inteligentes es como tener una computadora móvil, también la tecnología móvil cuenta con lo que es reproducciones de multimedia ya sea galería, reproductor de música, etc.

Según (Rojas y Ospina, 2012) nos menciona que el avance de la tecnología han hecho que estos aparatos incorporen funciones que no hace mucho parecían futuristas, como juegos, reproducciones de música mp3 y otros formatos, correo electrónico, SMS, agenda electrónica PDA, fotografía digital y video digital, video llamada, navegación por internet, GPS, y hasta televisión digital .Todo ha facilitado la comunicación entre las personas y hace que las labores diarias sean mucho más fáciles y rápidas de hacer.

Por lo tanto, podemos decir que en la actualidad contamos con una gran ayudad en entorno a nuestra vida para estar comunicados desde largas distancia la telefonía móvil es nuestra manera más fácil de estar comunicados. 
Al mencionar lo que nos permite realizar un dispositivo móvil estamos destacando los beneficios que tiene la telefonía móvil nos ofrece a nosotros como usuario ya que somos los primeros beneficiarios ya que contamos con un dispositivo móvil inteligente.

Los dispositivos móviles tienes sus respectiva clasificación y es importante destacar a muchas de ellas ya que nos mencionaba que los dispositivos son medio de comunicación y si son para estar comunicados con los demás ya sea de su vida o cualquier emergencia o a su vez son dispositivos muchas veces de entretenimiento ya sea de juegos o teniendo acceso a lo que es multimedia reproducción de música Mp3 etc.

\section{Importancia De La Tecnología En La Actualidad}

Según los autores (Leon, 2016), (Cordova, 2014) afirman que la tecnología es el conocimiento y la utilización de herramientas, técnicas y sistemas con el fin de servir a un propósito más grande como la resolución de problemas o hacer la vida más fácil y mejor, ya que es de gran importancia para los seres humanos ya ha ayudado y se ha adaptado a su ambiente.

Tener presente que la importancia de la tecnología ayuda a muchas personas a mantenernos comunicados ya que podemos cambiar nuestro entorno en muchas ocasiones no podemos dialogar personalmente pero hoy en día podemos hacer un video llamada y esto permite estar visualizando a la persona con la que se establece la comunicación por estos motivos debemos tener en cuenta lo importante que es la tecnología en nuestras vidas.

Por lo tanto, el desarrollo de la tecnología ha ayudado a conquistar las barreras de comunicación y reducir la brecha de la distancia entre la gente de todo el mundo, la tecnología es una colección de herramientas y conocimientos que hacen más fácil usar crear, administrar e intercambiar información. Gracias a la nueva tecnología, los lugares lejanos del mundo están conectados en cuestión de segundos.

\section{Características Generales De Los Dispositivos Móviles}

Según (Marco Galindo, y otros, 2010).Una gran cantidad de dispositivos electrónicos se clasifica actualmente como dispositivos móviles, desde teléfonos hasta tablets, pasando por dispositivos como lectores de RFID.Con tanta tecnología clasificada como móvil, puede resultar complicado determinar cuáles son las características de los dispositivos móviles.

A continuación, desarrollaremos las características esenciales que tienen los dispositivos móviles:

- Son aparatos pequeños.

- La mayoría de estos aparatos se pueden transportar en el bolsillo del propietario o en un pequeño bolso.

- Tienen capacidad de procesamiento. 
- Tienen conexión permanente o intermitente a una red.

- Tienen memoria (RAM, tarjeta MicroSD, flash, etc.).

- Normalmente se asocian al uso individual de una persona, tanto en posesión como en operación, cual puede adaptarlos a su gusto.

- Tienen una alta capacidad de interacción mediante la pantalla o el teclado.

En la mayoría de los casos, un dispositivo móvil puede definirse con cuatro características que lo diferencian de otros dispositivos, que puedan parecer similares. Estos cuatros características son:

\section{Movilidad}

Según los autores (Caraveo, 2012), (Marco Galindo, y otros, 2010) mencionan que la movilidad es la clave para que sus negocios siga funcionando de manera más ágil ya que el dispositivo móvil es la clave para el desarrollo y este tiene la capacidad de estar comunicados y poder acceder a información en cualquier momento y lugar, por lo tanto el dispositivo móvil es utilizado en cualquier momento gracias a la movilidad que este ofrece.

Es importante tener en cuenta que este dispositivo son lo suficiente pequeño para nosotros podemos llevar a cualquier parte sin importar nada porque es un aparato que no ocupa mucho espacio y además es liviano para llevarle a todas partes.

La movilidad para las personas es fundamental y que más con este dispositivo que nos facilita llevarle a toda parte como si fuera una computadora reducida ya que nos presta gran variedad de actividades que podemos realizar ya sea por su tamaño y por su manera de ser trasladado ya que este es la movilidad que necesitábamos para sentirnos más confiados al momento de estar comunicados.

\section{Tamaño reducido}

Según (Marco Galindo, y otros, 2010) Se entiende por tamaño reducido la cualidad de un dispositivo móvil de ser fácilmente usado con una o dos manos sin necesidad de ninguna ayuda o soporte externo. El tamaño reducido también permite transportar el dispositivo cómodamente por parte de una persona.

El tamaño es lo suficiente apropiado para el usuario tenga más movilidad y más adaptación a su tamaño ya que tiene un tamaño apropiado para llevar a cualquier parte asta en las manos, ya que el tamaño depende a la comodidad de cada persona muchas personas prefieren un tamaño un poco grande unos prefieren su tamaño normal el cual nos da más comodidad al momento de usarlo y realizar cualquier actividad en el dispositivo. 


\section{Comunicación Inalámbrica}

Según los autores (Marco Galindo, y otros, 2010), (Lopez, 2012) nos menciona que la comunicación inalámbrica es una trasferencia de información que se da en un dispositivo móvil ya sea para enviar o recibir información sin la necesidad de ningún cableado, sino que mediante a las ondas electromagnéticas a través del espacio.

La comunicación inalámbrica se destaca pos que no necesita ningún cable de andar cargando, sino que es utilizada a través de las ondas electromagnéticas esto hace que sea unan comunicación más ágil y segura sin necesidad de que se esté colgando o interrumpiendo o que la información llegue distorsionada.

A través de la comunicación inalámbrica se establecen más rápido sin necesidad de ningún cableado permitiéndonos así tener una comunicación más rápida, ágil y segura.

\section{Interacción Con Las Personas}

Según (Marco Galindo, y otros, 2010) nos menciona que el proceso de uso que establece un usuario con un dispositivo. Entre otros factores, en el diseño de la interacción intervienen disciplinas como la usabilidad y la ergonomía.

Este punto es muy importante destacar ya que si a través de la telefonía móvil podemos estar interactuando con más personas y realizar hasta videos llamada esto es lo que podemos decir que si se está interactuando porque también podemos transmitir alguna información para así estar comunicados y estar pendientes en lo que sucede a nuestro alrededor.

La interacción con las personas nos facilita al estar comunicados ya sea con personas que conocemos o con personas que estamos conociendo ya sea través de nuestras redes sociales permitiendo que este dispositivo móvil nos mantenga así conociendo personas a través de nuestra interacción de c conocer a más personas.

\section{Evolución Del Gobierno Electrónico Ecuatoriano.}

Según (Electronico, 2018-2021) A través de los años, una buena pauta para evidenciar el avance del gobierno electrónico mundial es el Índice de Desarrollo de Gobierno Electrónico (EGDI) de Naciones Unidas, este índice es el resultado de una medición que se efectúa cada dos años a sus 193 países asociados, en tres temáticas principales: servicios en línea, infraestructura de telecomunicaciones y capital humano (ver tabla 1). Para el año 2018 ubicó a Ecuador en el puesto 84 con un índice de 0,613 evidenciando con esto un crecimiento constante y colocándolo con este resultado sobre la media mundial y regional. 
Gráfico N 1. Índice desagregado EGDI 2010-2018, Ecuador

\begin{tabular}{|c|c|c|c|c|c|}
\hline \multirow{2}{*}{ (EGDI) İndice de Gobierno Electrónico } & 2010 & 2012 & 2014 & 2016 & 2018 \\
\hline & 0,433 & 0,487 & 0,505 & 0,563 & 0,613 \\
\hline \multicolumn{6}{|l|}{ Sub indices de Gobierno Electrónico } \\
\hline OSI (indice de servicios en linea) & 0,318 & 0.458 & 0,48 & 0,63 & 0,729 \\
\hline $\begin{array}{l}\text { Presencia Emergente } \\
\text { (Información básica en linea, publicación de políti- } \\
\text { cas, leyes, noticias) }\end{array}$ & 78 & 92 & 88 & 95 & \multirow{5}{*}{0,729} \\
\hline $\begin{array}{l}\text { Il Presencia Mejorada } \\
\text { (Facilidades para los usuarios, notificaciones, accesi- } \\
\text { bilidad web, acceso móvil, información de contacto, } \\
\text { seguridades) }\end{array}$ & 23 & 55 & 52 & 72 & \\
\hline $\begin{array}{l}\text { III Presencia Transaccional } \\
\text { (Servicios en linea: formularios en linea, ventanil- } \\
\text { la única de servicios, pago de servicios en línea, } \\
\text { trámites en linea) }\end{array}$ & 5 & 23 & 19 & 70 & \\
\hline IV Presencia Conectada & & & & & \\
\hline $\begin{array}{l}\text { (Interacción Ciudadano- Estado: chats, foros de dis- } \\
\text { cusión, participación electrónica) }\end{array}$ & 22 & 35 & 26 & 38 & \\
\hline TIII (Indice de Infraestructura de Telecomunicaciones) & 0,16 & 0,248 & 0,332 & 0,344 & 0,369 \\
\hline Abonados de telefonia móvil por cada 100 habitantes & 86,01 & 102,18 & 106,23 & 103,9 & 84,73 \\
\hline Abonados de telefonia fija por cada 100 habitantes & 14,17 & 14,41 & 14,9 & 15,28 & 14,96 \\
\hline $\begin{array}{l}\text { Abonados banda ancha inalámbrica por cada } 100 \\
\text { habitantes } \\
\text { (Se incluye este criterio desde 2014) }\end{array}$ & & & 21,62 & 26,7 & 46,93 \\
\hline Abonados banda ancha fija por cada 100 habitantes & 0,26 & 1,36 & 5,2 & 7,81 & 9,79 \\
\hline Uso de internet en la población (\%) & 9,71 & 24 & 35,13 & 43 & 54,06 \\
\hline $\mathrm{HCl}$ (Índice de Capital Humano) & 0,823 & 0,755 & 0,704 & 0.713 & 0,739 \\
\hline Tasa bruta de matricula (\%) & 78,52 & 82,08 & 75 & 83,73 & 88,87 \\
\hline Alfabetización en adultos (\%) & 84,2 & 84,21 & 91,59 & 94,46 & 94,35 \\
\hline $\begin{array}{l}\text { Media de años de escolaridad (Se incluyen este } \\
\text { criterio desde 2014) }\end{array}$ & & & 7,6 & 7,5 & 8,3 \\
\hline $\begin{array}{l}\text { Años esperados de escolaridad (Se incluyen este } \\
\text { criterio desde 2014) }\end{array}$ & & & 13,7 & 14,17 & 14 \\
\hline
\end{tabular}

Tabla 1: Indice desagregado EGDI 2010-2018, Ecuador

Fuente: (Electronico, 2018-2021)

Elaborado: Plan Nacional de Gobierno Electrónico 2018 - 2021 
Grafico N 1. Índice EGDI Ecuador desarrollo últimos 10 años, comparativo mundial y regional.

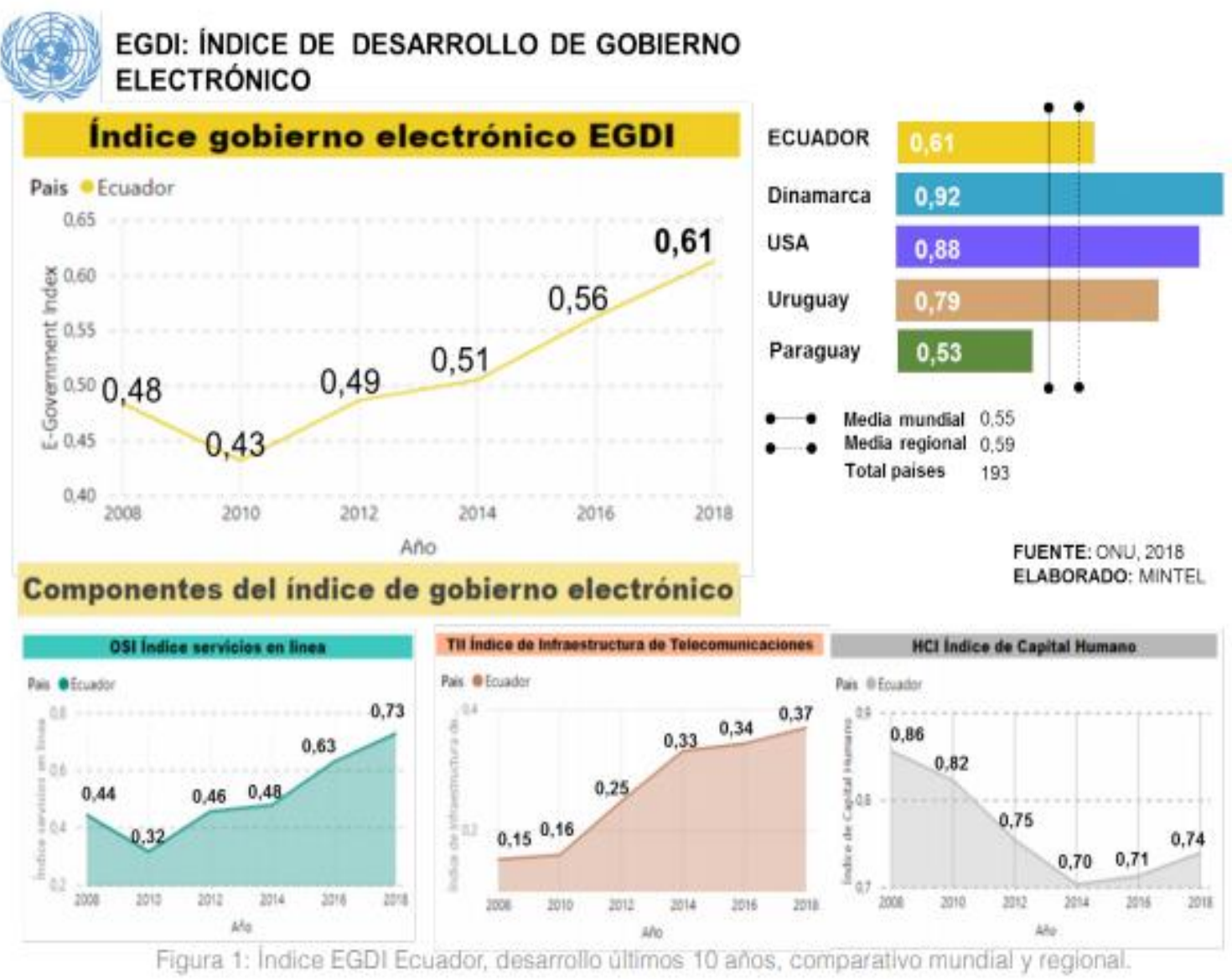

Fuente: (Electronico, 2018-2021)

Elaborado: Plan Nacional de Gobierno Electrónico 2018 - 2021

\section{Desarrollo De Las Telecomunicaciones En Ecuador}

Ecuador ha mostrado avances año a año y esto se puede observar en los resultados de la medición realizadas por naciones unidas en el E-GOVERNMENT SURVEY del 2018 donde se calificó a ecuador con un índice de 0,37 , valor que duplica al resultado obtenido en el 2010.

Este resultado se evidencia con mayor detalle en la información provista por la Agencia de Regulación y Control de las Telecomunicaciones Arcotel, donde señala que el 39\% de hogares en Ecuador posee internet fijo; existen 10,04 millones de cuentas de internet contratadas,(valor diez veces mayor respecto al año 2010); de estos 8,6 millones son cuentas de internet móvil y el 1,8 millones

de internet fijo (valor veinte veces mayor respecto al 2010). (Electronico, 2018-2021). 
Grafico N 2. Evolución de las telecomunicaciones en Ecuador 2010-2018

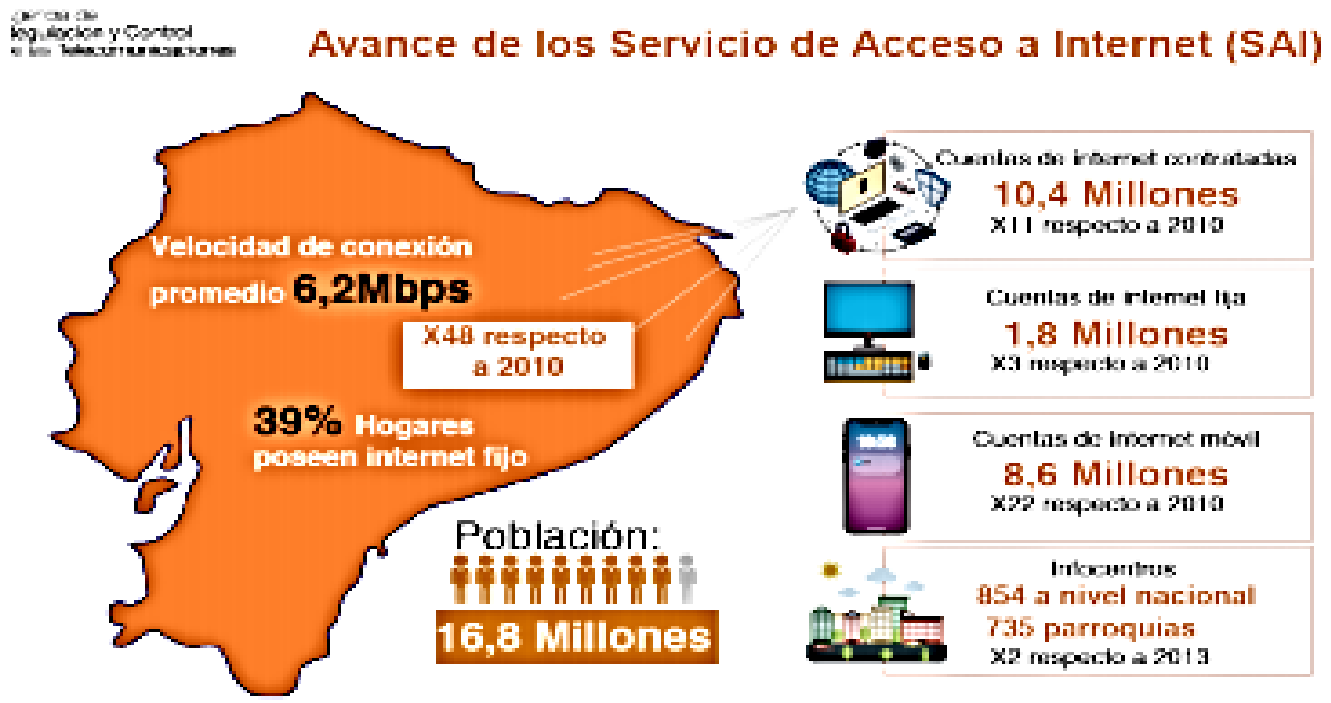

FUENTE: ARCOTEL, 2018 - TUENTE: AKAMAI, 2017 - ELABORADO: MINTEL.

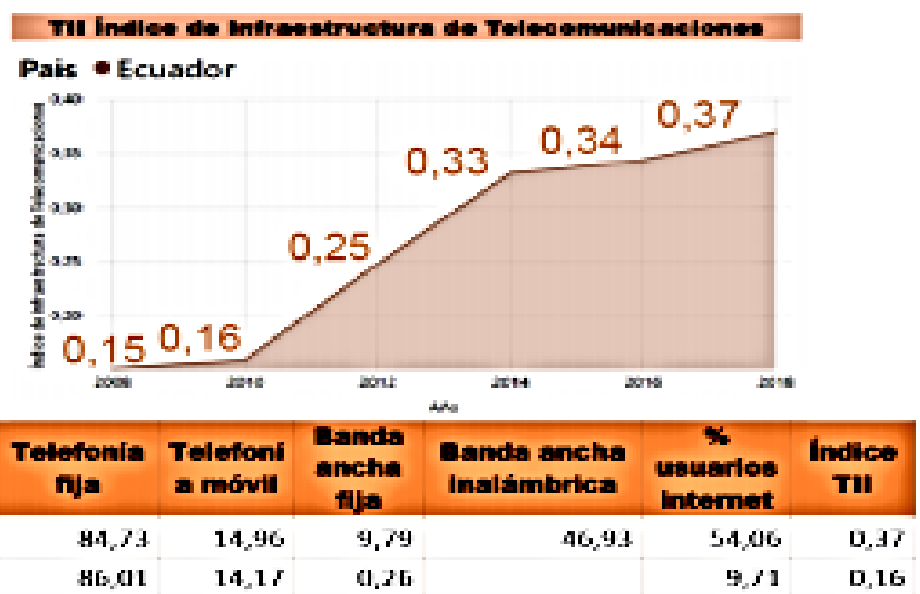

FUENTE: ONU - ELABOFADO: MINTEL

Figura 2: Evolución de las telocomunicaciones en Ecuador 2010-2018

Fuente: (Electronico, 2018-2021)

Elaborado: Plan Nacional de Gobierno Electrónico 2018 - 2021

El desarrollo de la telecomunicación ha sido tan notable en todo el aspecto poblacional ya que anteriormente pocas personas tenían acceso a un dispositivo móvil ya sea por escases económica etc.

Hoy en día en este nuevo mundo de nuevos conocimiento nuevas ciencia cada diez de cada ecuatoriano cuenta con esta herramienta muy fundamenta para la interacción de personas ya que antes se podía estar comunicados mediante cartas, hoy e mas fácil ya sea a través de mensajes de texto de voz o estar comunicados por las redes sociales por esto Arcotel nos refleja una muy interesante información acerca de cuantas personas e habitante estamos haciendo uso de la telefonía móvil o fija ya que hace varios año esto ha sido un crecimiento muy grande para las telecomunicaciones facilitándole a ellos que 
la red de comunicación cada ves de amplié mucho más así ellos tienen una mayor tasa de crecimiento ya sea nacional e internacionalmente.

\section{El Ecuador Y Usos De Teléfonos Fijos O Celulares Móvil.}

Según (Àvalos, 2015) nos menciona que los datos del Instituto de Estadísticas y Censos (INEC) se menciona que el $81,7 \%$ de los hogares posee al menos un teléfono celular, 8.2 puntos más que lo registrado en el 2009.

Grafico N 1. Gráfico de Teléfonos Fijos O Celulares Móvil.

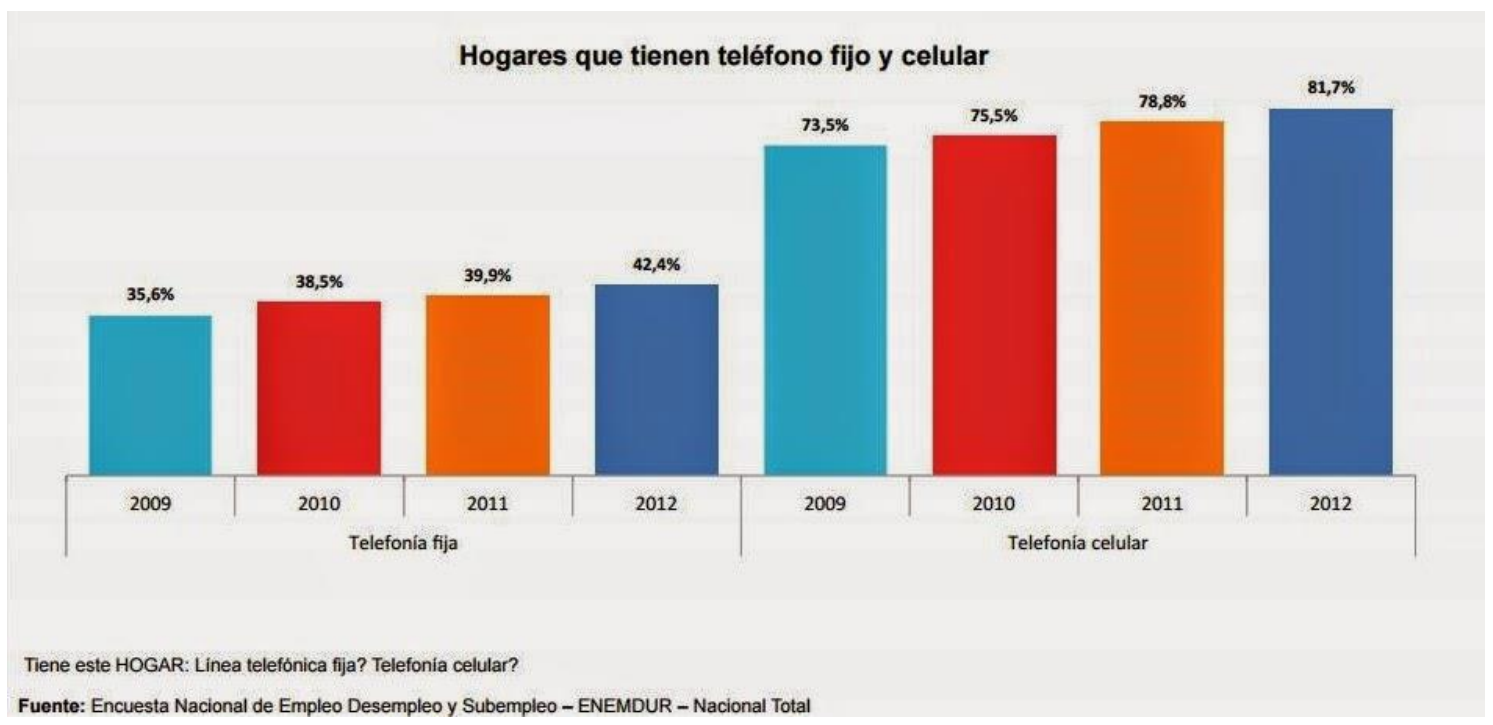

Fuente: (Àvalos, 2015)

Elaborado: Comunicación Multimedia 2015.

Nos menciona avalos que se da a través del censo fue que se obtuvieron estos datos estadísticos ceso que se aplicó en el año 2009 y como actualmente nos e ha establecido un censo para saber la actualidad de cuanto han incrementado estos datos ya que hoy la telefonía móvil se ha evolucionado más que en aquellos tiempos ya que tenemos variedades de marcas y modelos que se hay hoy en día y cada vez se ha incrementado lo que es el celular ya que cada una de las personas usamos más el celular ya que este se puede trasladar a todas partes a diferencia de lo que es teléfono fijo.

\section{Crecimiento De La Telefonía Fija Móvil Beneficia A Todos Los Ecuatorianos}

Según (Informacion) el avance tecnológico durante las últimas décadas, genero diversos cambios en el Ecuador, las áreas afines al sector de las telecomunicaciones colaboran vital importancia en el manejo de la comunicación directa entre los ciudadanos, y el desarrollo económico, político, cultural y financiero del país.

En el 2006, en telefonía fija existían 1'748.049 líneas que beneficiaban a los hogares ecuatorianos. El informe de rendición de cuentas del Ministerio de Telecomunicaciones y de la Sociedad de la Información de 2012 indica que existen 2`331.925 líneas telefónicas fijas .Cada día que transcurre ene le Ecuador, hace que los ecuatorianos tengan 
mayor posibilidad de comunicarse a través de las líneas telefónicas ya sea para comunicarse con sus familiares o para realizar intercambios comerciales.

El crecimiento de las telefonía móvil e tan notable que cada vez es más grande la cifras de incrementación de personas hogares que cuentan con lo que son telefonía fija ya que esta esta forma más ágil para estar comunicados por medio de estos dispositivos ya que son de grandes ayudas en nuestra vida a que según el portafolio menciona que nosotros realizamos varias actividades en el dispositivo móvil ya sea en el entorno laboral o familiar.

La telefonía móvil cada vez está en crecimiento ya que más por el adolecente estos prefieren estar o tener un celular dependiendo de cuál este a la moda cada vez el consumo se va incrementando por eso las cifras de que hemos observado anteriormente son bajas para las que creo que si se hace serán el triple de las que han habido en el año 2012. Estos también a nosotros como ecuatorianos nos facilitan ya que cada vez el dispositivo móvil se vuelve más indispensable en nuestras vidas.

\section{La Telefonía Móvil Y Su Impacto Ambiental En Ecuador}

Según (Another, 2010).Los teléfonos hoy en día son muy utilizados ya que brindan una comunicación más ágil pero al pasar los años esto empieza los problemas los teléfonos cuando son desechados por lo general este va con su batería la misma que es muy dañina para el medio ambiente ya que son una fuente muy potencial de contaminación por sus metales obtenidos en el primer orden conforme los avances se están dando es muy común que se desechen rápidamente.

La telefonía como menciona Another es de gran ayuda y si cuando el dispositivo a nosotros con usuarios que hacemos uso de esto dispositivos cuando nos comienza a fallar lo desechamos y por muchas razones no tenemos en cuenta que le estamos haciendo daño a nuestro medio ambiente, muchas veces por estar al día con las nuevas marcas o modelos desechamos un dispositivo que a veces pueda que esté funcionando muy bien sin embargo nosotros por prejuicios de estar al día en el mundo de la moda tecnológica no tenemos en cuenta cuánto daño nos estamos haciendo a nosotros mismo y a nuestro entorno que nos rodea.

\section{Ventajas Y Desventajas De La Telefonía Móvil En La Sociedad}

Según (Dutta, 2016) nos menciona las siguientes ventajas y desventajas de la telefonía móvil son las siguientes:

Gráfico N 1. Gráfico de ventajas y desventajas de la telefonía móvil en la sociedad.

\begin{tabular}{|c|c|}
\hline Ventajas & Desventajas \\
\hline $\begin{array}{l}\text { - La función de la comunicación } \\
\text { inalámbrica es la mejor ventaja } \\
\text { que ofrece un teléfono móvil. La } \\
\text { capacidad de comunicarse por }\end{array}$ & $\begin{array}{l}\text { - Problema de salud hay muchos } \\
\text { estudios para los efectos negativos } \\
\text { de salud del uso incesante del } \\
\text { teléfono móvil la infertilidad }\end{array}$ \\
\hline
\end{tabular}


voz, textos, incluso correos electrónicos han hecho posible la interacción en cualquier momento de humano a humano a través de vasta de áreas geográficas.

- El teléfono móvil puede ser utilizado para cualquier emergencia tales como médicas, desastres relacionados con el clima, accidentes o delitos.

- Las aplicaciones corporativas las industrias, empresas han aprovechado la nueva herramienta de la nueva era y las capacidades de las aplicaciones basadas en los teléfonos inteligentes y en otros teléfonos móvil de alta tecnologías. masculina el efecto de la radiación de microondas en el cerebro y los patrones de sueño irregulares en los preadolescentes se han convertido en unos de los principales problemas del uso de los teléfonos móviles.

- Los problemas de estas expresiones no son ya no son exclusivas de los mensajes de texto sino que han llegado al ámbito educativo como apuntes, exámenes etc.

\section{Fuente: (Dutta, 2016)}

Elaborado por: Solórzano María

Las ventajas como lo mencionamos anteriormente son de gran importancia tener en cuenta ya que se podría decir que nos mantiene comunicados ante cualquier situación y las desventajas son destacadas porque así como ofrece ventaja también tenemos desventajas ya que la telefonía móvil en ocasiones son perjudiciales para nuestro entorno ya que es un medio de distracción para las personas aunque en gran parte hemos visto que se ha producido la gran parte de accidentes de tránsito han sido ocasionados por estar distraído en el celular.

\section{El Avance De La Telefonía Ofrece Soluciones Corporativas}

Según (Portafolio, 2016) En la actualidad, las telecomunicaciones son uno de los sectores más importantes para cualquier país, ya que además de contribuir al desarrollo económico y social, mejoran la calidad de vida de la población.

Por eso es que las empresas se ven obligadas a incluir estas herramientas para agilizar sus procesos internos y externos so empleadas, cliente, respectivamente, además de facilitar y estimular la comunicación entre la parte.

Precisamente los celulares inteligentes son la herramienta preferida por las personas para desarrollar dichas tareas, al punto de que este dispositivo ha llegado a convertirse casi en una extensión de nuestro cuerpo con el cual ejecutamos una gran cantidad de tareas de manera más ágil y práctica 
Este avance tecnológico podemos observar que se ha evolucionado muy satisfactoriamente tanto para la vida social como en la vida laboral ya que cuando estamos en una empresa muchas veces asemos acceso a nuestro dispositivo móvil para realizar cualquier tarea encomiendas tenemos que tener presente que son unas herramientas de gran ayuda ya que son unos dispositivos livianos y pequeño de su tamaño y esto agilita su movilidad.

\section{Conclusiones}

- Con la información recolectada sobre el avance de la tecnología móvil es de gran importancia conocer y saber las ventajas y desventajas que tienes estas herramientas ya que este dispositivo nos permite realizar varias actividades.

- La telefonía móvil nos permite estar comunicados desde cualquier parte de mundo.

- Las características de un dispositivo móvil son muy adecuadas para el gusto de la ciudadanía ya que son dispositivo pequeño, livianos nos permite estar cómodo con sus variedades de uso y sus funciones que cumple.

- $\quad$ El impacto que produce en la ciudadanía la telefonía móvil es muy favorable ya que se utiliza estos dispositivos ya en el aspecto laboral o familiar.

\section{Referencias Bibliográficas}

Another, Just. 2010. Impacto ambiental que producen los celulares. [En línea] Enero de 2010. [Citado el: 19 de Octubre de 2018.] https://gpencalada.wordpress.com/2010/01/13/impactos-ambientales-queproducen-los-celulares-pilas-y-focos-ahorradores/.

Àvalos, Valeria. 2015. Comunicación Multimedia 2015. [En línea] 15 de Mayo de 2015. http://comunicacionmultimedia2015.blogspot.com/2015/05/consumo-y-uso-delas-tics-en-el-ecuador.html.

Caraveo, Alfonso. 2012. Tecnologia más movilidad:binomio clave en logistica. Logistica. [En línea] 07 de Julio de 2012. [Citado el: 13 de Octubre de 2018.] www.logisticamx.enfasis.com/articulos/64532-tecnologia-mas-movilidadbinomio-clave-en-logistica.

Cordova, Daniel. 2014. La importancia de la tecnologia en la actualidad. Prezi. [En línea] 29 de Marzo de 2014. [Citado el: 19 de Octubre de 2018.] http://prezi.com/9-anlamdtrx/la_importancia_de_la_tecnologia_en_la_actualidad/?click_source=logge d_element\&page_location=header\&element_type=link\&element_text=pricing.

Diseño, Libro blanco para la telefonia movil acesible y facil de usar. 2012. [En línea] Diciembre de 2012. [Citado el: 19 de Octubre de 2018.] http://www.amovil.es/sites/default/files/e- 
5_1_libro_blanco_espanol.pdf?fbclid=IwAR28nqCO2S7LcFlr3nSaLzq70GpsN Oh_FpV-CO6nn9HQL741CHC2-DhPMas.

Dutta, Pallab. 2016. Ventajas y desventajas de telefonos moviles. Techlandia. [En línea] 2016. [Citado el: 19 de Octubre de 2018.] https://techlandia.com/ventajasdesventajas-telefonos-moviles-hechos_39242/.

Electronico, Plan Nacional de Gobierno. 2018-2021. [En línea] 2018-2021. [Citado el: 18 de Octubre de 2018.] https://www.gobiernoelectronico.gob.ec/wpcontent/uploads/2018/09/PNGE_2018_2021sv2.pdf.

Garita, Raul Alberto. 2013. Tecnologia Movil:desarrollo de sistemas y aplicacion para las unidades de Informacion. s.l. : E-Ciencias de la Informacion, 2013. pág. 15. Vol. 3. ISSN-1659-4142.

Informacion, Ministerio de Cominicaciones y de la Sociedad de la. Crecimiento de la telefonia fija movil beneficia a todos los Ecuatorianos . [En línea] [Citado el: 20 de Octubre de 2018.] https://www.telecomunicaciones.gob.ec/crecimiento-de-latelefonia-fija-y-movil-beneficia-a-todos-los-ecuatorians/.

Leon, Dr.Edgar. 2016. La Importancia de la Tecnologia. [En línea] 17 de Abril de 2016. [Citado el: 13 de Octubre de 2018.] htt://ahoranews.net/La-importancia-de-latecnologia/.

Lopez, Rafael. 2012. Comunicacion Inalambrica . Ing.Disp moviles . [En línea] 15 de Febrero de 2012. [Citado el: 19 de Octubre de 2018.] ingeneria-dispositivosmoviles.blogspot.com/2012/12/tecnologias-inalambricas.html.

Marco Galindo, Maria Jesús, y otros. 2010. Escaneando la informática. Barcelona : UOC, 2010. 978-84-9788-110-4.

Portafolio. 2016. El avance de la telefonia movil ofrece soluciones corporativas . [En línea] Noviembre de 2016. [Citado el: 23 de Octubre de 2018.] https://www.portafolio.co/innovacion/el-avance-de-la-telefonia-movil-ofrecesoluciones-corporativas-501781.

Quijada, Lisbeth. 2014. Avances tecnologicos de telefonos celulares. [En línea] 02 de Abril de 2014. [Citado el: 18 de Octubre de 2018.] avancestecnologicosenlatelefoniamovil/blogspot.com.

Rojas y Ospina, 2012. 2012. Impacto de la tecnologia movil en la sociedad. Santiago : s.n., 2012. pág. 3.

Weiss Muller, Michelle. 2008. Avance tecnologicos de celulares. Buenos Aires, Argentina : s.n., 2008. Vol. 19. 1668-5229. 


\section{Para citar el artículo indexado.}

Velasteguí López, E., \& Barona López, G. (2019). El avance en la tecnología móvil y su impacto en la sociedad. Explorador Digital, 2(4), 5-19. https://doi.org/10.33262/exploradordigital.v2i4.337

\section{Ciencia \\ LDigital}

El artículo que se publica es de exclusiva responsabilidad de los autores y no necesariamente reflejan el pensamiento de la Revista Explorador Digital.

El articulo queda en propiedad de la revista y, por tanto, su publicación parcial y/o total en otro medio tiene que ser autorizado por el director o editor de la Revista Explorador Digital.

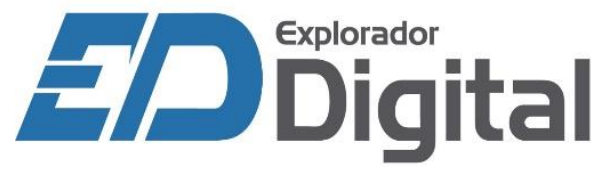

